\title{
Analysis of Clinicopathological Features and Prognostic Factors in 39 Cases of Bladder Neuroendocrine Carcinoma
}

\author{
HUI-HUI ZHOU ${ }^{1 *}$, LI-YAN LIU ${ }^{2 *}$, GUO-HUA YU ${ }^{1}$, GUI-MEI QU ${ }^{1}$, \\ PEI-YOU GONG ${ }^{1}, \mathrm{XIAO}_{\mathrm{YU}^{3}}$ and PING YANG ${ }^{1}$ \\ Departments of ${ }^{1}$ Pathology and ${ }^{3}$ Medicine Yuhuangding Hospital of Qingdao University, Yantai, P.R. China; \\ ${ }^{2}$ Department of Medicine, The fifth People's Hospital of Jinan, Jinan, P.R. China
}

\begin{abstract}
Aim: Through analysis and summarization of clinicopathological features, immunohistochemical expression, pathological diagnostic criteria, prognostic and other factors in patients suffering from bladder neuroendocrine carcinoma (BNEC), a better understanding of BNEC could be achieved to provide solid evidence for clinicopathology and prognosis. Materials and Methods: The clinicopathological data of 39 cases of BNEC with up to 5-year follow-up data (median follow-up=650 days) were analyzed retrospectively based on immunohistochemical staining. Survival analyses were carried out using the Kaplan-Meier method and tested with the logrank method. Multivariate Cox regression analysis was adopted to screen independent risk factors affecting patients' survival. In these 39 cases of BNEC, there were 26 cases of male patients, 13 female, with the proportion of male to female being 2:1. The ages of onset ranged from 44 to 86 , with the median age being 62 and the average age 61.97 years, respectively. Histologically, referring to the WHO standard of neuroendocrine lung tumor classification, there were 7 cases of typical carcinoid tumors, 8 atypical carcinoid, 12 small-cell carcinomas and 12 large-cell carcinomas. In these cases there were 11 cases of featured urothelium carcinomas and 9 cases of adenocarcinomas. Results: Immunohistochemical staining showed that, in these 39 cases of BNEC, the positive expression for the neuroendocrinic markers, including neural cell adhesion
\end{abstract}

\footnotetext{
*These Authors contributed equally to this study.

Correspondence to: Xiao Yu, MD and Ping Yang, Departments of Pathology and Medcine, Yuhuangding Hospital of Qingdao University, 20\# Yuhuangdingdong Road,Yantai 264000, Shandong Province, P.R. China. Tel: +86 6691999-86269, Fax: +86 6691999 , e-mail: 13356997292@163.com and gpy_6681258@163.com
}

Key Words: Bladder, NEC, pathological features, immunohistochemistry. molecule 56 (CD56), synaptophysin (Syn), chromogranin A $(\mathrm{CgA})$, neuron-specific enolase (NSE), thyroid transcription factor-1 (TTF-1), cytokeratin (CK) and cytokeratin 7 (CK7), accounted for 39/39, 27/39, 18/39, 39/39, 19/39, 10/39 and $8 / 39$, respectively. In contrast, cytokeratin 20 (CK20), protein 63 (P63), human melanoma black 45 (HMB45), S-lfln protein 100 (S-100) and leukocyte common antigen (LCA) were all negatively expressed. During the follow-up period, 12 patients died. The 1-, 3- and 5-year overall survival (OS) rates were $76.92 \%, 74.36 \%$ and $69.23 \%$. Conclusion: BNEC is one of the most malignant tumors with severe invasiveness and poor prognosis. Immunohistochemistry revealed that CD56, Syn, CgA, NSE, TTF-1, CK, CK7, CK20, P63, HMB45, S-100 protein and LCA immune markers play important roles in diagnosis and differentiation. Many factors, including the patient's age, size and shape of the tumor, operative method, perineuronal invasion, vascular invasion, distant organ metastasis and pathological type, show great difference in influencing OS time of patients, among which the size of the tumor, no invasion, vascular invasion and distant organ metastasis are independent risk factors affecting prognosis (survival time). Radical cystectomy is the prior alternative to treat this tumor.

Neuroendocrine tumors refer to the heterogeneous tumors stemming from peptidergic neurons and neuroendocrine cells in the diffuse neuroendocrine system, which may occur in organs and tissues in any part of the body (1). These tumors are likely to carry-out multi-potential differentiation secreting various active hormones, thus showing significant differences in biological behaviors and prognosis. They mostly appear in the lungs and digestive tract (2), rarely in the bladder. Thus, clinical, pathological and follow-up data were collected from 39 cases of bladder neuroendocrine carcinoma (BNEC) after diagnosis in the Department of Pathology between 2000 and 2015. Based on the data collected, understanding of BNEC can be deepened so that solid bases are set for clinical treatment and prognostic judgment. 


\section{Materials and Methods}

Materials. The 39 cases of BNEC originate from large specimens externally examined at Yantai Affiliated Hospital of Binzhou Medical University Hospital, the 5th People's Hospital of Jinan, Yantai Yuhuangding Hospital and the Affiliated Hospital of Qingdao University from January 2000 to December 2015. All patients in these 39 cases had not undergone radiotherapy or chemotherapy prior to surgery. Postoperatively, 3 chief physicians jointly examined the radiographs under the microscope and drew their conclusions. Clinical data and summaries were collated from the medical record room and sample inspection sheet in the respective Hospitals, while the followup data were obtained by telephone interview or in the household registration Department of the local Public Security Bureau.

Methods. The samples from the 39 cases of BNEC were fixed with $10 \%$ neutral buffered formaldehyde, dehydrated and embedded in paraffin. The immunohistochemical streptavidin-peroxidase (SP) method was adopted, with primary antibodies being neural cell adhesion molecule 56 (CD56), synaptophysin (Syn), chromogranin A (CgA), neuron-specific enolase (NSE), thyroid transcription factor1 (TTF-1), cytokeratin 7 (CK7), CK20, human melanoma black 45 (HMB45), S-lfln protein 100 (S-100), leukocyte common antigen (LCA) and 3,3'-diaminobenzidine (DAB) for staining All reagents were purchased from Zhongshan Biotech Co. Ltd., Beijing, China. NSE, CK, Syn, CgA, CK7, CK20 and HMB45 stain positively in the cytoplasm, S-100 protein stains positive in the cytoplasm and nucleus, LCA and CD56 stain positive in the membrane, whereas protein 63 (P63) stains positively in the nucleus.

Follow-up. In this study, all 39 cases of BNEC were followed up by phone and with the household registration Department of the local Public Security Bureau. Another 28 cases were excluded due to lack of available follow-up data. Follow-up data enabled us to discern the survival time and the patients' outcomes. The duration of the research started from the confirmation of diagnosis by the respective Pathology Department to the death of the patients or the end of the study.

Statistical analysis. Data analysis was performed using statistical software SPSS17.0. (http://www.spss.com) The enumeration data were compared with the $\chi^{2}$ test. Survival analyses were analyzed using the Kaplan-Meier method and tested with the log-rank method. Multivariate Cox regression analysis was adopted to screen independent risk factors affecting the survival of patients, with differences being statistically significant when $p<0.05$.

\section{Results}

Clinical data. In these 39 cases of BNEC, 26 cases were males and 13 females, with the proportion of male to female ratio being $2: 1$. The age of onset ranged from 44 to 86 years with a median age of 62 and average age of 61.97 , respectively. In 24 cases, patients were over the age of 60 years. There was no statistical significance between the males' and the females' age of onset $(p=0.819)$. Nineteen of the 39 cases showed tumors on the left lateral wall, 12 on right lateral wall, 2 at right parietal area, 4 on right posterior wall and 2 in the bladder neck. Ultrasonics, in most cases, revealed a thickened bladder wall, which was the site of the occupying lesion (Figure 1A). The clinical symptoms of 25 cases featured hematuria in part, if not the whole course of the study. In these 25 cases, 15 patients described pain and/or discomfort in the waist and/or abdomen. Ten cases showed urinary irritation symptoms, whilst 4 cases had no significant symptoms upon physical examination. The course of illness varied from 1 day to 2 years. Only in 12 cases did the patients smoke. Of the 26 cases that were subjected to radical total cystectomy treatment, 5 cases had partial cystectomy and 8 cases transurethral resection of the bladder tumor.

Pathological features. Nine of the 39 cases of BNEC showed the shape of a mushroom, 1 polypoida, 26 ulcer and 3 lobular. The diameters of tumors ranged from $0.8 \mathrm{~cm}$ to $13 \mathrm{~cm}$, with the median diameter being $3 \mathrm{~cm}$ and average diameter 3.42 $\mathrm{cm}$, respectively. There were 13 cases of vessel invasion, 10 cases with no invasion and 11 cases of distant organ metastasis. Referring to the WHO standard of neuroendocrine lung tumor classification (3), there were 7 cases of typical carcinoid (Figure 1B), 8 cases of atypical carcinoid (Figure

Figure 1. Hematoxylin and eosin (HE) morphological and immunohistochemical phenotype. A: Low-echo convex on the left lateral wall of the bladder with wide basal part and rough surface. B: Typical carcinoid with tumor cells of identical shape, mitotic count less than 2/10 high-power fields (HPFs). HE slides magnified under low-power lens show organoid, trabecular, island-like or palisade arrangement, without necrosis. C: Atypical carcinoid with tumor cells of the same morphology, mitotic count less than 2 10/10 HPFs. HE slides magnified under lowpower lens show organoid, trabecular or island-like arrangement, with necrosis. D: Small-cell carcinoma with small tumor cells of oat-like, egglike shapes with little cytoplasm and round or egg-like nucleus. HE slides magnified under medium power lens show chromatin of fine grain shapes with no clear nucleoli but obvious mitotic count and extensive necrosis. E: Large-cell carcinoma with tumor cells of organoid, palisade or rosette arrangement. HE slides magnified under medium power lens show large tumor cells with cytoplasm of medium size, clear nucleoli, obvious mitotic count and extensive necrosis. F: Both small-cell carcinoma and urothelium carcinoma in HE slides magnified under low-power lens. G: Both large-cell carcinoma and adenocarcinoma in HE slides magnified under low-power lens. H: Small-cell carcinoma with neural cell adhesion molecule 56 (CD56)-positive tumor cells, magnified under medium-power lens with EnVision. I: Small-cell carcinoma with synaptophysin (Syn)positive tumor cells, magnified under medium-power lens with EnVision. $J$ : Small-cell carcinoma with chromogranin A (CgA)-positive tumor cells, magnified under medium-power lens with EnVision. K: Small-cell carcinoma with neuron-specific enolase (NSE)-positive tumor cells, magnified under medium-power lens with EnVision. L: Small-cell carcinoma with thyroid transcription factor-1 (TTF-1)-positive tumor cells, magnified under medium-power lens with EnVision. M: Small-cell carcinoma with cytokeratin (CK)-positive tumor cells, magnified under medium-power lens with EnVision. N: Both small-cell carcinoma and urothelium carcinoma appear with CK7-positive tumor cells of urothelium carcinoma, magnified under medium-power lens with EnVision. 


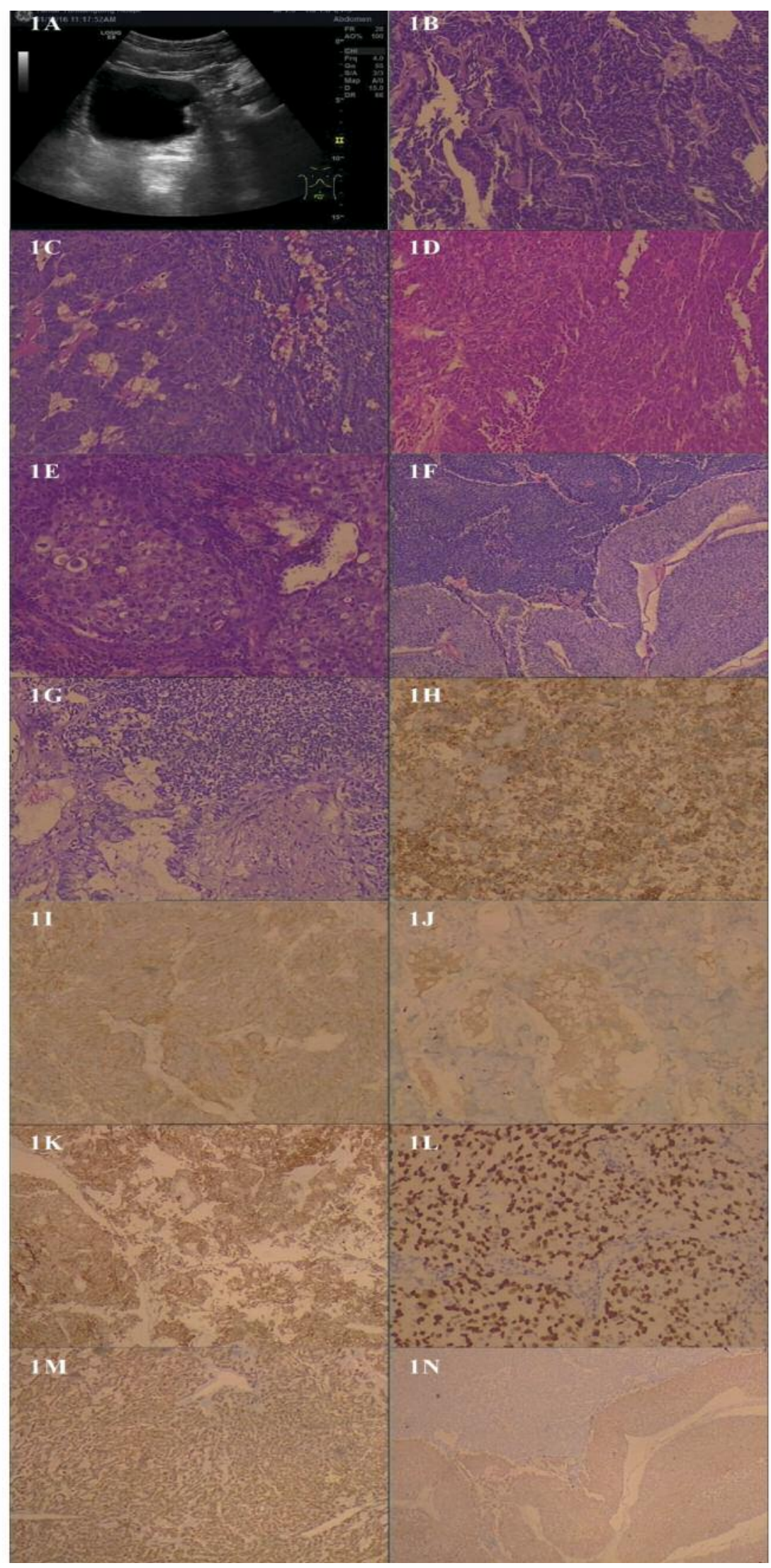



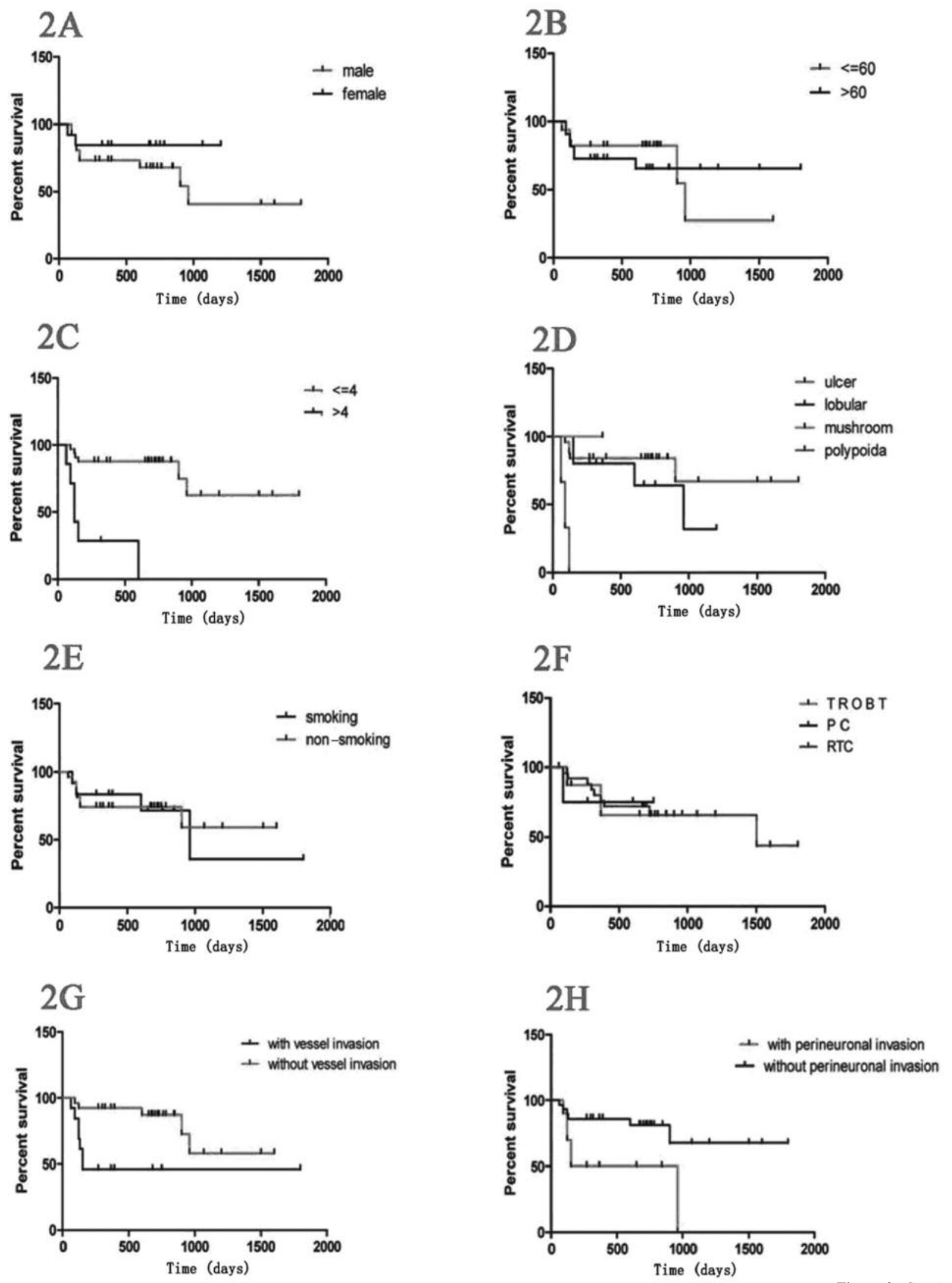

Figure 2. Continued 

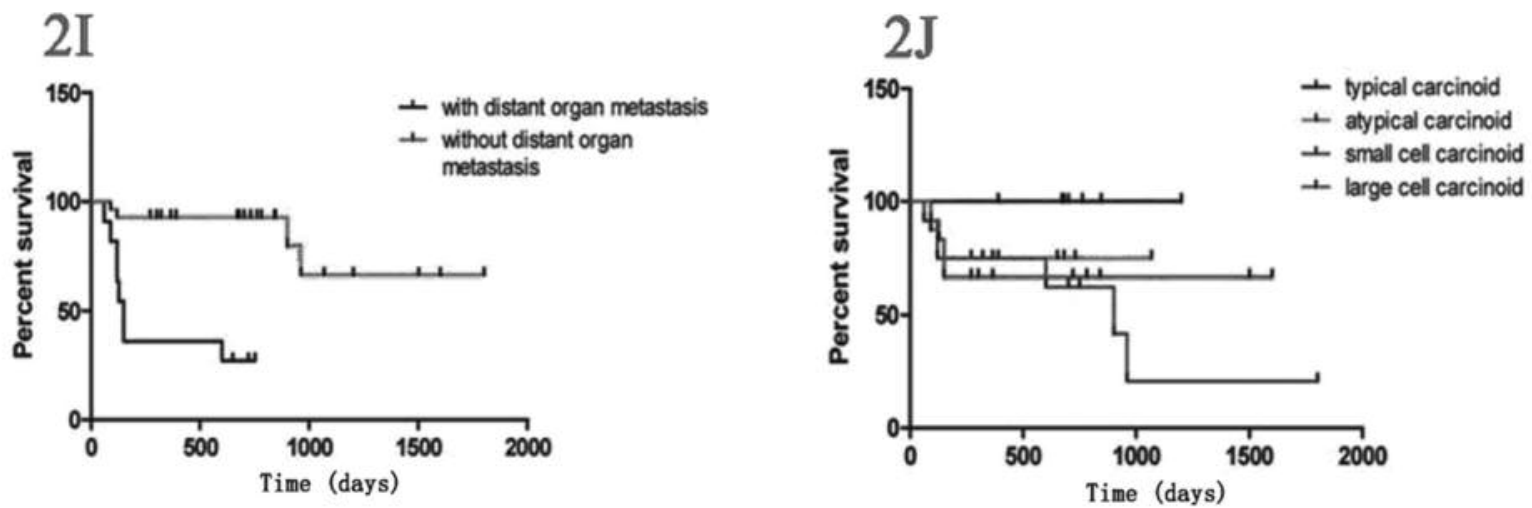

Figure 2. Survival curves of bladder neuroendocrine carcinoma (BNEC) patients for different pathological parameters. A: Overall survival between different genders was not statistically significant ( $p=0.227)$; B: Overall survival among different ages showed significant difference ( $p=0.013)$; : Overall survival between different sizes of tumor was statistically significant ( $p=0.001) ; D$ : Overall survival between different shapes of tumor was statistically significant ( $p=0.001) ;$ E: Overall survival between smokers and non-smokers had no significant difference ( $p=0.867) ; F: O v e r a l l$ survival among transurethral resection of bladder tumors (TROBT), partial cystectomy (PC) and radical total cystectomy (RTC) ( $p=0.026)$; G: Overall survival between different vessel invasion with statistical difference ( $p=0.001) ; H$ : Overall survival between different nerve invasion had statistically significant differences ( $p=0.016)$; I: Overall survival between different distant organ metastases was statistically significant ( $p=0.001$ ); J: Overall survival among typical carcinoid, atypical carcinoid, small-cell carcinoma and large-cell carcinoma pathological types was statistically significant $(p=0.027)$.

1C), 12 cases of small-cell carcinoma (Figure 1D) and 12 cases of large-cell carcinoma (Figure 1E). Among them, 11 cases featured urothelium carcinoma (Figure 1F) and 9 cases adenocarcinoma (Figure 1G). According to the tumor-nodemetastasis (TNM) classification of bladder cancer, there were 15 cases of phase I, 4 cases of phase II, 2 cases of phase III and 18 cases of phase IV cancer.

Immunohistochemical staining. The positive expression of 39 cases of BNEC for the neuroendocrine markers included CD56 (Figure 1H), Syn (Figure 1I), CgA (Figure 1J), NSE (Figure 1K), TTF1 (Figure 1L), CK (Figure 1M) and CK7 (Figure 1N) were 39/39, 27/39, 18/39, 39/39, 19/39, 10/39 and $8 / 39$, respectively. In contrast, CK20, P63, HMB45, S100 protein and LCA were all negative.

The relationship between clinicopathological features and prognosis. In this study, only 39 cases had follow-up data from the day of diagnosis to December 31st, 2015. The follow-up lasted from 60 days to 1,800 days, with median follow-up lasting 650 days. In this period, 12 patients died with survival time averaging 290.58 days. The 1-, 3- and 5year overall survival (OS) rates were $76.92 \%, 74.36 \%$ and $69.23 \%$, respectively.

The effect of gender on survival rate. In the 39 cases of BNEC, there were 26 male patients (66.67\%) 10 (38.46\%) of whom died. In the 13 female patients (33.33\%), 2 $(15.38 \%)$ died. Differences in survival rates between different genders were not statistically significant over the whole survival period $(p=0.227)$ (Figure 2A).

The effect of age on survival rate. In the 39 cases of BNEC, there were $15(38.46 \%)$ cases in which patients were younger than 60 years of age, $4(26.67 \%)$ of which died during the course of this study. There were $24(61.44 \%)$ cases with patients over the age of 60 years, 8 (33.33\%) of which died. Differences in survival rate between these two age groups was considered statistically significant in the OS period $(p=0.013)$ (Figure 2B).

The effect of the sizes of the tumors on survival rate. In the 39 cases of BNEC, there were $25(64.10 \%)$ cases in which tumor size was $\leq 4 \mathrm{~cm}$. Of these 25 patients, $4(16 \%)$ died. Tumors larger than $4 \mathrm{~cm}$ appeared in $14(35.90 \%)$ cases, of which 8 (57.14\%) patients died. Differences in survival rate between these two tumor size groups was considered statistically significant in the OS period $(p=0.001)$ (Figure 2C).

The effect of the shape of the tumor on survival rate. In the 39 cases of BNEC, $26(66.67 \%)$ cases showed the shape of an ulcer, of which 6 patients died. Of the 9 cases with mushroom-like tumors, 3 patients $(33.33 \%)$ died. There was $1(2.56 \%)$ case of polypoida in which the patient survived the follow-up period. Three (7.69\%) cases of the 39 featured a lobular shape; of these patients, all $3(100 \%)$ died. Survival rate differences between the differently shaped tumors was considered statistically significant over the course of the follow-up period $(p=0.001)$ (Figure 2D). 
The effect of smoking on survival rate. In the 39 cases of BNEC, 12 (30.77\%) patients were smokers, of which 4 $(33.33 \%)$ died. Twenty seven patients $(69.23 \%)$ had no history of smoking, of which $8(29.63 \%)$ died. The differences in survival rates between these two groups did not reach statistical significance during the follow-up period $(p=0.867)$ (Figure 2E).

The effect of operative methods on survival rate. In the 39 cases of BNEC, $8(20.51 \%)$ cases underwent transurethral resection of their bladder tumors, of which $2(25 \%)$ patients died. There were $5(12.82 \%)$ patients who underwent partial cystectomy, of whom 1 (20\%) died. Twenty six (66.67\%) patients underwent radical total cystectomy, with $9(34.62 \%)$ dying. Differences in survival rates among transurethral resection of bladder tumors, partial cystectomy and radical total cystectomy were considered statistically significant during the follow-up period ( $p=0.026$ ) (Figure 2F).

The effect of vessel invasion on survival rate. In the 39 cases of BNEC, 13 (33.33\%) cases had vascular invasion, of which $7(53.85 \%)$ patients died. There were $26(66.67 \%)$ cases in which there was no evidence of vascular invasion, with 9 $(34.62 \%)$ patients dying. Differences in survival rates between these two groups, of vascular invasion did not reach statistical significance during the follow-up period $(p=0.001)$ (Figure 2G).

The effect of no invasion on survival rate. In the 39 cases of BNEC, $10(25.64 \%)$ cases had no invasion; of these 10 patients, $4(40 \%)$ died. No sign of perineuronal invasion appeared in 29 cases $(74.36 \%)$; however, 8 patients (27.59\%) died. Differences in survival rates between these two groups were statistically significant over the total follow-up period $(p=0.016)($ Figure 2H)

The effect of distant organ metastasis on survival rate. In the 39 cases of BNEC, $11(28.20 \%)$ cases had distant organ metastasis, with 8 (72.23\%) patients dying. There were 28 (71.79\%) cases in which there was no sign of distant organ metastasis but, nevertheless, 4 (14.29\%) patients died. Differences in survival rates between these two groups were statistically significant in total survival during the follow-up period $(p=0.001)$ (Figure 2I).

The effect of pathological types on survival rate. In the 39 cases of BNEC, there were 7 (17.95\%) cases of typical carcinoid with no deaths noted in the follow-up period. Eight (20.51\%) patients had a typical carcinoid; 2 (25\%) died. Of the $12(30.77 \%)$ cases of small-cell carcinoma, $6(50 \%)$ patients died. There were $12(30.77 \%)$ cases of large-cell carcinoma where 4 patients $(33.33 \%)$ died. Differences in survival rates among typical carcinoid, atypical carcinoid
Table I. Multivariable Cox regression analysis of overall survival.

\begin{tabular}{lcc}
\hline Variables & HR $(95 \% \mathrm{CI})$ & $p$-Value \\
\hline $\begin{array}{l}\text { Tumor size } \\
\leq 4 \mathrm{~cm}\end{array}$ & 1.01 & 0.001 \\
$\quad>4 \mathrm{~cm}$ & $(1.47-6.93)$ & \\
Vessel invasion & 5.92 & 0.002 \\
$\quad$ Yes & $(1.57-22.3)$ & \\
$\quad$ No & 5.47 & \\
Never invasion & $(1.35-22.0)$ & 0.034 \\
$\quad$ Yes & & \\
$\quad$ No & 26.60 & 0.001 \\
Distant organ metastasis & $(5.98-118.2)$ & \\
$\quad$ Yes & \\
No &
\end{tabular}

HR, Hazard ratio; CI, confidence interval.

small-cell carcinoma and large-cell carcinoma were statistically significant ( $p=0.027$ ) (Figure $2 \mathrm{~J}$ ).

Results of prognostic multivariate analyses. Multivariable Cox regression analysis selecting principal variables revealed that the size of the tumor, perineuronal invasion, vascular invasion and distant organ metastasis are independent risk factors affecting the prognosis of the BNEC patients (survival time). Factors are shown in Table I.

\section{Discussion}

Epidemic investigations show that NEC in bladder is quite rare, only accounting for $0.5-1.0 \%(3,4)$ of all malignant tumors in the bladder, with no specificity $(5,6)$ in clinical manifestation and imaging features. Up to now, there is no confirmed reason for BNEC. It has been reported both domestically and abroad that BNEC is closely related to smoking (7-9). However, in relation to the 12 smokers included in our study, no statistical differences in survival rates between smokers and non-smokers were seen during the 5-year follow-up period. In order to solve this puzzle, we need to enlarge the scale of sampling to further investigate the role of smoking in BNEC. The most vulnerable group of people for BNEC tends to be older men, with the main clinical manifestation being painless gross hematuria. Some patients also had symptoms of dysuria, frequency and urgency of micturition and urodynia. BNEC mostly appears on the lateral walls, followed by posterior and back walls. Under cystoscope, tumors had mushroom, polypoida, ulcer or lobular shape (6). Concerning gender, age, clinical symptoms and tumor location, there were 26 male and 13 female cases, with those over 60 accounting for 24 out of the 39 cases $(61.54 \%)$. The clinical symptoms of 25 cases featured gross hematuria in part, if not the whole, of the 
course. In these 25 cases, patients in 15 cases suffered from pain in the waist or (and) abdomen. Ten cases showed urinary irritation symptoms, while 4 cases had no significant symptoms upon physical examination. Nineteen out of the 39 cases showed tumors on the left lateral wall, 12 on right lateral wall, 2 at right parietal area, 4 on right posterior wall and 2 in bladder neck. Nine of the 39 cases of BNEC showed mushroom, 1 polypoida, 26 ulcer and 3 lobular shape. All of these equate with reports in the literature (9).

Diagnosis of BNEC mainly depends on histopathology, immunohistochemistry and ultrastructural features, similar to NEC in the lung and other tissues (10-12). NEC in large cells of the bladder is fairly rare, with only 85 case-reports up to now. In these 85 cases, 35 simple and 50 mixed-type cases have been reported. Little is known about the clinical data of BNEC. In our study, 10 of the 12 cases had mixed features with urothelium carcinoma and adenocarcinoma. Immunohistochemistry staining convincingly suggests that diagnosis of BNEC, with other frequently used immunohistochemical markers, should be performed with the use of Syn, CgA, CD56 and NSE markers. Alijo et al. (11) have reported 44 cases of small-cell carcinoma of the urinary bladder and 2 cases of large-cell neuroendocrine carcinoma (LCNEC). Of them, small-cell carcinoma positively expressed Syn (50\%), CgA (30\%), CD56 (68\%), NSE (80\%) and TTF-1 (100\%). As shown in our data, the 12 cases of small-cell carcinoma positively expressed CD56 (12/12), Syn (11/13), CgA (8/12), CK17 and TTF-1 (9/13) to different degrees. This result revealed that CD56, Syn and CgA show greater positive rates than previously stated in the literature. In our 12 reported cases of LCNEC, 3 neuroendocrine markers, including Syn, CgA and CDA56, were all positively expressed (12/12). This is different from the literature mentioned above (11) and may be attributed to the clonal code of the antibodies used. Further studies are required with a larger scope of sampling. TTF-1 is a specific marker of thyroid and pulmonary tumors. According to our data, 19 out of 24 cases of the BNEC positively expressed TTF-1, including 11 cases of small-cell carcinoma and 8 cases of large-cell carcinoma. These markers cannot be used as an index of identification for metastatic small-cell carcinoma in the lung. Instead, clinical data and other immune markers should be considered along with comprehensive analyses in the laboratory.

In view of the special prognostic significance, it is important to identify the following diseases in diagnosis. Firstly, high-grade urothelium carcinoma has obvious pleomorphic cells of large size and rich cytoplasm. They have a lightly-stained cytoplasm and immunohistochemical staining shows specificity of tumor cells expressing CK7 with no expression of neuroendocrine markers, such as CD56, Syn and $\mathrm{CgA}$. The second to be identified is lymphoma. Malignant lymphoma, which is primarily present in bladder, is rarely seen. When the tumor cell is very small (small-cell carcinoma), it is hard to distinguish. Immunohistochemistry of malignant lymphoma expresses specific lymphocyte markers (CD3, CD20, LCA and so on) without expression of neuroendocrine and epithelial markers (CK or epithelial membrane antigen (EMA)), which is helpful for identification. In this group of cases, CD3, CD20 and LCA were all negative. Thirdly, malignant melanoma also requires attention. Primary malignant melanoma of the bladder is very rare and mostly metastatic. Histologically, tumor cells present polygonal and fusiform shape with clearly pleomorphic nuclei. The nucleolus is large and clear with mitotic count, which is easily seen. Immunohistochemistry of tumor cells reveals S-100 protein, HMB45 and Melan A, which are identifiable. In our cases, S-100 protein, HMB45 and Melan A were all negative. Fourthly, chemodectoma or pheochromocytoma need to be distinguished. Chemodectoma often occurs in bladder, especially in women, clinically manifested by 3 typical symptoms, namely persistent or idiopathic sudden hypertension, intermittent gross hematuria and micturition seizure. The patients suffer from paroxysmal headaches, shivering, sweating and syncope. Histologically, tumor tissue displays, in the nest type, rich interstitial blood vessels. Tumor cells are round or polygonal of the same size with few Sertoli cells around the nest. Although immunohistochemistry of tumor cells reveals presence of Syn and $\mathrm{CgA}$, differentiation could be ascertained by the negative staining of CK-pan and EMA. Sertoli cells feature positive S100 protein staining. In our group of cases, S-100 was negatively expressed, which was helpful in differentiation. Fifthly, lymphoepithelioma-like carcinoma (LELC) needs to be mentioned. Tumor cells show flaky or nest-like shapes of different sizes. The size of tumor cells is very large, with bubbly nuclei. The nucleolus is very clear with abundant lymphocytes and plasma cells. In this work, immunohistochemistal staining of tumor cells showed only epithelial markers, without expression of neuroendocrine markers, which can be used for identification. Sixthly, metastatic small-cell carcinoma is another topic to be discussed. There are clinical records showing malignant tumors in other body parts. When urothelium carcinoma features appear histologically, it helps the diagnosis of the primary tumor. What is noticeable is that BNEC can also express TTF-1, as is shown in 19 of the 39 cases presented in this report. Therefore, this marker cannot be used for the differentiation of primary and metastatic tumors. The seventh disease that needs to be distinguished from BNEC is prostatic small-cell carcinoma invading the bladder. Three cases of small-cell carcinoma of the urinary bladder invading prostate are included in this work, which are very similar to this seventh disease, without obvious differences in immunophenotyping. Therefore, it is hard to differentiate these two distinct cases. We consider that the pathogenic sites 
are found mainly in the bladder, with urothelium carcinoma features included in tumor tissue, while there is only a small focus of small-cell carcinoma within the prostate. This is helpful to the diagnosis of primary small-cell carcinoma of the bladder. Recently, scholars have discovered that the TMPRSS2-ERG fusion gene is unique to the prostate and also highly expressed in prostatic small-cell carcinoma. Schelling et al. (13) and Wang et al. (14) have reported that the positive rates of TMPRSS2-ERG fusion gene in 54 cases and 61 cases of prostatic small-cell carcinoma are $48 \%$ (26/54) and $54 \%$ $(33 / 61)$, respectively, by testing with fluorescence in situ hybridization, which provides another new method of differentiation between the two and, therefore, can be adopted to identify metastatic prostatic small-cell carcinoma. The eighth disease to be mentioned is neuroblastoma. Due to its small tumor cell size and rarefactive cytoplasm, it is hard to distinguish neuroblastoma from small-cell carcinoma. However, neuroblastoma stems from original neuroepithelial cells, mostly retroperitoneal, especially adrenal. It often affects infants, with the bladder being the least common place to be found. Microscopy shows that the tumor tissue has a lobular or nest-like shape with vague boundaries. There are lightly stained neurofibrils of unequal amounts between tumor cells. Immunohistochemically, these cells express neuroendocrine markers, including Syn, CgA, etc. but do not express epithelial markers. The histological origin of BNEC is still not clear with vivid debate around this issue. Currently, most scholars consider it to originate from stem cells of multiple differentiation potentials within the bladder. This can also be used to explain the vast heterogeneity of BNEC, for which various neuroendocrine and epithelial markers can be expressed. It often coincides with urothelium carcinoma, squamous cell carcinoma and adenocarcinoma (14). In our 39 cases of BNEC, there were 11 with urothelium carcinoma and 9 with adenocarcinoma, supporting the result of the origin of pluripotent stem cells differentiating in different directions (15). Cheng et al. (16) have also proved that small-cell carcinoma of the urinary bladder and urothelium carcinoma originate from the same histocyte.

According to published reports and their follow-up data, BNEC is a type of malignant tumor with strong invasive behavior and bad prognosis. In some cases, patients present with metastasis or have entered advanced stages (12) when diagnosed. All 39 cases featured obvious bladder wall infiltration on pathological examination (18 cases with infiltration of muscularis propria, 21 with infiltration of outer membrane and external adipose tissue of outer membrane). Survival analysis showed that the age of the patient, size and shape of the tumor, perineuronal invasion, vascular invasion, distant organ metastasis and pathological types have different effects on the OS of patients. Multivariable Cox regression analysis, selecting principal variables, revealed that the size of the tumor, never invasion, vascular invasion and distant organ metastasis are independent risk factors affecting the prognosis of the patient (survival time). Without other reports available, either domestic or international, further studies with enlarged sampling should be carried out to exclude the effect of limited sampling and ensure the reliability of this research. For treatment, most scholars suggest combining radical bladder dissection and chemotherapy to improve clinical effectiveness. Of course, there are also some scholars adopting neoadjuvant chemotherapy, which has been shown to have good efficacy (4). In recent years, the development of molecular targeted therapy for malignant tumors has advanced and some investigators suggest that molecular targeted therapy of BNEC using epidermal growth factor receptor (EGFR), B-cell lymphoma 2 (Bcl-2) and CD56 might hold promise, potentially bringing significant improvement to the survival rate of BNEC patients (17).

\section{References}

1 Oberg K and Castellano D: Current knowledge on diagnosis and staging of neuroendocrine tumors. Cancer Metastasis Rev 30(Suppl 1): 3-7, 2011.

2 Kim JH, Lee SH, Park J, Kim HY, Lee SI, Nam EM, Park JO, Kim K, Jung CW, Im YH, Kang WK, Lee MH and Park K: Extrapulmonary small-cell carcinoma: A single-institution experience. Jpn J Clin Oncol 34: 250-254, 2004.

3 Trias I, Algaba F, Condom E, Espanol I, Segui J, Orsola I, Villavicencio H and Garcia Del Muro X: Small cell carcinoma of the urinary bladder. Presentation of 23 cases and review of 134 published cases. Eur Urol 39: 85-90, 2001.

4 Ahsaini M, Riyach O, Tazi MF, El Fassi MJ, Farih MH, Elfatmi $\mathrm{H}$ and Amarti A: Small cell neuroendocrine carcinoma of the urinary tract successfully managed with neoadjuvant chemotherapy. Case Rep Urol 2013: 598325, 2013.

5 Kim JC, Kim KH and Jung S: Small cell carcinoma of the urinary bladder: CT and MR imaging findings. Korean J Radiol 4: 130-135, 2003.

6 Tunc B, Ozguroglu M, Demirkesen O, Alan C, Durak H, Dincbas FO and Kural AR: Small cell carcinoma of the bladder: A case report and review of the literature. Int Urol Nephrol 38: 15-19, 2006.

7 Abrahams NA, Moran C, Reyes AO, Siefker-Radtke A and Ayala AG: Small cell carcinoma of the bladder: A contemporary clinicopathological study of 51 cases. Histopathology 46: 57-63, 2005.

8 Cheng L, Pan CX, Yang XJ, Lopez-Beltran A, MacLennan GT, Lin H, Kuzel TM, Papavero V, Tretiakova M, Nigro K, Koch $\mathrm{MO}$ and Eble JN: Small cell carcinoma of the urinary bladder: A clinicopathologic analysis of 64 patients. Cancer 101: 957962, 2004.

9 Ismaili N, Elkarak F, Heudel PE, Flechon A and Droz JP: Small cell cancer of the bladder: The Leon-Berard cancer centre experience. Indian J Urol 24: 494-497, 2008.

10 Alijo Serrano F, Sanchez-Mora N, Angel Arranz J, Hernandez C and Alvarez-Fernandez E: Large cell and small cell neuroendocrine bladder carcinoma: immunohistochemical and outcome study in a single institution. Am J Clin Pathol 128: 733-739, 2007. 
11 Travis WD, Brambilla E, Nicholson AG, Yatabe Y, Austin JH, Beasley MB, Chirieac LR, Dacic S, Duhig E, Flieder DB, Geisinger K, Hirsch FR, Ishikawa Y, Kerr KM, Noguchi M, Pelosi G, Powell CA, Tsao MS, Wistuba I and Panel WHO: The 2015 World Health Organization Classification of Lung Tumors: Impact of genetic, clinical and radiologic advances since the 2004 classification. J Thorac Oncol 10: 1243-1260, 2015.

12 Varinot J, Colin P, Roupret M, Leroy X and Comperat E: Pathologic analysis of upper tract urothelial carcinomas: State of the art review for the yearly scientific report of the French National Association of Urology. Prog Urol 24: 954-965, 2014.

13 Schelling LA, Williamson SR, Zhang S, Yao JL, Wang M, Huang J, Montironi R, Lopez-Beltran A, Emerson RE, Idrees MT, Osunkoya AO, Man YG, Maclennan GT, Baldridge LA, Comperat $\mathrm{E}$ and Cheng L: Frequent TMPRSS2-ERG rearrangement in prostatic small cell carcinoma detected by fluorescence in situ hybridization: The superiority of fluorescence in situ hybridization over ERG immunohistochemistry. Hum Pathol 44: 2227-2233, 2013.

14 Wang L, Williamson SR, Zhang S, Huang J, Montironi R, Davison DD, Wang M, Yao JL, Lopez-Beltran A, Osunkoya AO, MacLennan GT, Baldridge LA, Du X and Cheng L: Increased androgen receptor gene copy number is associated with TMPRSS2-ERG rearrangement in prostatic small cell carcinoma. Mol Carcinog 54: 900-907, 2015.
15 Hata S and Tasaki Y: A case of the large cell neuroendocrine carcinoma of the urinary bladder. Case Rep Med 2013: 804136, 2013.

16 Cheng L, Jones TD, McCarthy RP, Eble JN, Wang M, MacLennan GT, Lopez-Beltran A, Yang XJ, Koch MO, Zhang S, Pan CX and Baldridge LA: Molecular genetic evidence for a common clonal origin of urinary bladder small cell carcinoma and coexisting urothelial carcinoma. Am J Pathol 166: 1533-1539, 2005.

17 Walker BF, Someren A, Kennedy JC and Nicholas EM: Primary carcinoid tumor of the urinary bladder. Arch Pathol Lab Med 116: 1217-1220, 1992.
Received May 2, 2017

Revised May 25, 2017

Accepted May 26, 2017 